\title{
Déterminants Socio-Économiques De L'adoption Des Technologies Agro Écologiques De Productions Agricoles Dans Le Département De Mayahi Au Niger
}

\author{
Mahamane Moctar Rabe, \\ Université de Tahoua, Niger, \\ Ibrahim Baoua, \\ Université Dan Dicko DanKoulodo de Maradi, Niger, \\ Rabo Issaka Salissou \\ Cabinet Sahel bio de Maradi, Niger
}

Doi:10.19044/esj.2021.v17n43p73

Submitted: 29 September 2021

Accepted: 24 November 2021

Published: 31 December 2021
Copyright 2021 Author(s)

Under Creative Commons BY-NC-ND 4.0 OPEN ACCESS

Cite As:

Rabe M.M. „Baoua I.,\& Salissou I. R.,(2021). Déterminants Socio-Économiques De L'adoption Des Technologies Agro Écologiques De Productions Agricoles Dans Le Département De Mayahi Au Niger European Scientific Journal, ESJ, 17(43), 73.

https://doi.org/10.19044/esj.2021.v17n43p73

\section{Résumé}

Cette étude conduite dans le département de Mayahi, au Niger oriental, a pour objectif, d'identifier les facteurs influençant l'adoption des pratiques culturales de production du mil et du niébé et aussi celle de l'utilisation du bloc multi-nutritionnel. Ainsi, cent-vingt (120) chefs d'exploitation choisis aléatoirement ont été enquêtés sur l'utilisation des technologies de production diffusées au cours des trois dernières années. La régression logistique a permis de déterminer les corrélations entre les contextes et l'adoption des technologies. Ainsi, l'implication des producteurs dans les démonstrations a influencé positivement sur l'adoption des techniques d'application du compost ( $\mathrm{P}<0,001)$, l'utilisation des variétés améliorées de mil et de niébé $(\mathrm{P}<0,05)$, des extraits aqueux des graines de Neem $(\mathrm{P}<0,001)$ et de la technologie de stockage hermétique des récoltes $(\mathrm{P}<0,001)$. Cependant, la proximité des villages du marché hebdomadaire a eu une corrélation négative avec l'utilisation du compost $(\mathrm{P}<0,05)$; alors que la proximité du village aux zones urbaines a influencé positivement l'achat des blocs multi nutritionnels densifiés et la pratique du défrichement amélioré $(\mathrm{P}<0,001)$. Par ailleurs, l'accès au crédit a influencé positivement sur l'achat des blocs multi 
nutritionnels densifiés $(\mathrm{P}<0,05)$; le contact avec les services de vulgarisation agricole public a été corrélé par l'utilisation de la technologie de lutte contre la chenille mineuse de l'épi du mil $(\mathrm{P}<0,05)$. Ces résultats peuvent contribuer à orienter les acteurs de développement rural à mieux planifier les activités de diffusion et de promotion des nouvelles pratiques agronomiques.

Mots clés: Adoption, bloc multi-nutritionnel, Logit, Niébé, Mil, Niger, Afrique de l'Ouest

\title{
Determinants of Agroecological Technologies Adoption in Mayahi The Department, Niger
}

\author{
Mahamane Moctar Rabe, \\ Université de Tahoua, Niger, \\ Ibrahim Baoua, \\ Université Dan Dicko DanKoulodo de Maradi, Niger, \\ Rabo Issaka Salissou \\ Cabinet Sahel bio de Maradi, Niger
}

\begin{abstract}
The objective of this study is to identify the determinants of production technology's adoption of millet and cowpea as well as the densified multinutritional block in the department of Mayahi. One hundred and twenty (120) randomly selected farm managers were surveyed on the use of production technologies released over the past three years. Logistic regression was used to determine that were between contexts and technology adoption. Results showed that producers' participation in the demonstration, the adoption of compost application techniques, the use of improved varieties of millet and cowpea, aqueous extracts and neem seeds, and hermetic crop storage technology positively influenced. Similarly, results reveal that the proximity of villages to the weekly market had a negative correlation with the use of compost and the location of the village next to urban areas positively influenced the purchase of densified multi-nutritional blocks. $\mathrm{R}$ ults also indicate that the practice of improved land clearing and access to credit positively influenced the purchase of densifieal blocks; while contact with public agricultural extension services was correlated with the use of millet ear leaf miner control technology. These findings could help to guide rural development agents to better plan activities for the dissemination and promotion of new agronomic practices.
\end{abstract}

Keywords: Adoption, Logit, Niébé, Mil, Niger 


\section{Introduction}

En Afrique de l'Ouest, l'agriculture contribue pour 30\% au PIB et emploie plus de $50 \%$ de la population active (Renard et al., 2004). Ainsi, pour Jama \& Gonzalo (2008), le développement agricole se trouve au cœur des stratégies de réduction de la pauvreté et de la sécurité alimentaire. Au Niger, les trois quart de la population vivent dans la partie Sud, soit $25 \%$ de la superficie du territoire . La principale source d'activité économique du pays est liée à la production agro-sylvo-pastorale. Ces activités occupent plus de $80 \%$ de la population active. En effet, elle représente près d'un quart du Produit Intérieur Brut (PIB) et emploie environ 70\% de la population (Banque Mondiale, 2010). Le défi de l'agriculture nigérienne demeure celui d'augmenter la production pour répondre à la croissance des besoins alimentaires et aussi d'assurer des revenus des populations pour éliminer la pauvreté en milieu rural. Comme toutes les agricultures du monde, et à l'exception de quelques complexes agricoles et agro-industriels de type capitaliste, l'agriculture se pratique sur des petites exploitations agricoles familiales (EAF) sans recours à la mécanisation d'où son caractère archaïque et traditionnel. Les EAF jouent un rôle important dans la vie socioéconomique des populations et constituent la principale source de subsistance (Barmo et al., 2017). Cependant, cette agriculture se trouve confrontée à plusieurs contraintes dont la faible utilisation des variétés améliorée (Ibro \& Bokar, 2001; Sani \& Bagna, 2007) ; la pression croissante des insectes ravageurs ; la pauvreté des sols qui est l'une des plus importantes contraintes qui limitent le potentiel de l'agriculture (Serme et al., 2015) et le faible encadrement des producteurs et l'inaccessibilité aux innovations agricoles (MAG-SDR. 2010).

Plusieurs stratégies ont été développées avec les producteurs par la mise en place des champs de démonstration de nouvelles technologies pour l'augmentation des rendements et des revenus. La présente étude a été conduite dans une grappe de vingt villages qui ont reçu un encadrement sur l'utilisation des technologies dites agro-écologiques dans la période 2014 - 2017 par le projet d'appui à la sécurité alimentaire par l'intensification agro-écologique dans le département de Mayahi. Il a été démontré des innovations pour la gestion de la fertilité des sols, la lutte contre les ennemis des cultures et l'alimentation animale tout en faisant abstraction à l'utilisation des engrais et pesticides chimiques qui sont des méthodes peu accessibles aux producteurs, mais aussi néfastes à l'environnement.

L'objectif principal de ce travail est de connaître les facteurs influençant l'adoption des pratiques culturales de production du mil et du niébé mais aussi celle de l'utilisation du bloc multi-nutritionnel densifié dans le département de Mayahi. 


\section{Matériel Et Méthodes}

\subsection{Matériel}

\section{Zone de l'étude}

Elle couvre les communes de Mayahi, Attantané, Sherkin haoussa et Tchaké dans le département de Mayahi qui est situé dans la partie Nord-Est de la région de Maradi. Il s'agit des villages dans lesquels le projet d'appui à la sécurité alimentaire par l'intensification agro-écologique intervient depuis 2014 pour la vulgarisation d'un paquet des technologies agro-écologiques (Semences améliorées, compost, RNA, zai, culture en bandes alternées, biopesticides, lutte biologique, sacs PICS) à travers la conduite des champs de démonstration. Ces activités s'étendent sur quatre communes (Sherkin Haoussa, Mayahi, Attantané, et Tchaké) avec 5 villages chacun, soit 20 villages au total. Le climat est de type sahélien avec une saison de pluie de juin à octobre et une saison sèche de novembre à mai. La pluviométrie moyenne annuelle calculée en 2015 est de 450 mm (MA, 2015). Les sols sont de type sablonneux et sablo-argileux. L'étude a concerné tout producteur cultivant le mil et le niébé et résidant dans le village de l'étude.

\section{Echantillonnage}

Les enquêtes ont concerné 6 des 20 villages, soit 30\% des concernés par l'implantation du processus de champ de démonstration au cours des trois années d'exécution du projet $(2014,2015,2016)$. Le choix de ces villages a été fait de façon aléatoire. Par village, 20 producteurs ont été enquêtés dont 10 ont été impliqués dans la mise en œuvre des technologies agro écologiques et les 10 autres étant des producteurs du village mais non directement concernés par les démonstrations. Au total, sur les 120 producteurs formés à travers les champs de démonstration, 60 ont été enquêtés de façon aléatoire, soit $50 \%$ de l'effectif des formés et 60 autres producteurs témoins ont été aussi choisis aléatoirement et ajoutés au formés.

Le questionnaire individuel a permis d'obtenir des données quantitatives et qualitatives sur les caractéristiques sociodémographiques des producteurs, les connaissances des technologies, leur décision d'adoption ou non de ces technologies. Ces données ont été collectées à travers l'application «KoBocollect » intégrée dans des tablettes. Le choix de cette application s'explique par son accès gratuit sur internet et sa facilité d'usage.

D’après Sherin et al. (2018), KoBoToolbox est une entrée des données en ligne gratuite et open source outil est développé par l'initiative humanitaire de Harvard avec le soutien de diverses organisations comme Brigham et Women's Hospital, USAID. 


\section{Technologies agro-écologiques}

Il a été démontré au cours des 3 années $(2014,2015,2016)$ avec un groupe de 20 producteurs par villages les technologies suivantes: le défrichement amélioré, le labour profond pour l'ameublissement du sol, l'apport de la fumure organique ou du compost pour le redressement de la fertilité du sol, le Zaï pour limiter les pertes d'eau par ruissellement dans les champs, les biopesticides à base de graines de Neem pour la gestion des insectes ravageurs du niébé ; la lutte biologique avec les lâchers augmentant le parasitoïde de Habrobracon hebetor pour la lutte contre la mineuse de l'épi du mil, le sac PICS (Purdue Improved Cowpea Storage) pour la conservation sans pesticide du niébé et la fabrication des blocs multi nutritionnels densifiés pour la valorisation des résidus des cultures et l'amélioration de la production animale. Ces technologies constituent des variables dépendantes qui sont influencées par d'autres variables indépendantes de nature socio-économique. Selon Houndekon \&Gogan (1996) plusieurs facteurs sont susceptibles d'influencer l'adoption d'une pratique agronomique. Ici, il est analysé des facteurs suivants : (1) le genre. Selon Dey (1981), les hommes ont plus accès à l'information et aux intrants comparativement aux femmes, ce qui accroit la probabilité d'adoption des technologies agricoles en leur faveur. Il est donc émis l'hypothèse que cette variable influence positivement la probabilité d'adopter une nouvelle technologie chez les producteurs ; (2) l'âge et ansexpag où l'expérience des producteurs en agriculture pourrait favoriser l'adoption des nouvelles technologies. Selon Adesina (1996), les paysans possédant une longue expérience ont le temps de pratiquer les technologies et de découvrir leur apport positif ; (3) l'éducation où le niveau d'instruction peut être une variable considérée comme déterminante dans l'adoption des technologies agricoles. Dans cette étude, est considéré comme instruit, tout individu qui a fréquenté une classe d'école formelle ou d'école informelle donc celui qui sait au moins lire ou écrire en français ou en anglais. Il accroît le sens de l'innovation, l'habileté et la facilité d'apprécier des nouvelles technologies (Adéoti et al., 2002). Cette variable pourrait influencer positivement l'adoption de la technologie. La variable contaagri (4) permet d'estimer l'influence du contact avec les structures de recherche et de vulgarisation. L'approche participative est considérée comme un élément indispensable dans l'adoption des nouvelles technologies (Neuchâtel, 1995). Il est donc émis l'hypothèse qu'être apprenant dans un champ de démonstration pourrait influencer positivement la décision du producteur à adopter les technologies agricoles proposées. L'appartenance à une Organisation des Producteurs est une variable binaire qui prend la valeur 1 si le producteur est membre d'une OP et 0 dans le cas contraire. Tout membre d'une OP est informé et formé sur les nouvelles technologies ou innovations. L'hypothèse selon laquelle l'adhésion des producteurs à une OP influence l'adoption des technologies reste à vérifier. Le 
problème de ressources financières est une réalité chez les petits producteurs donc l'accès au crédit favorise l'adoption de nouvelles technologies. La proximité du marché au village est une variable binaire qui mesure la distance du village au marché périodique le plus proche. Elle prend la valeur 1 pour les villages proches d'un marché périodique $(<10 \mathrm{~km})$ et avec une route facilitant l'accès, et 0 pour les villages éloignés. La facilité d'accès au marché peut être un facteur influençant positivement l'adoption de nouvelles technologies agricoles (Sanginga et al., 1999) car elle est susceptible d'améliorer la qualité du produit à vendre. Donc il y a lieu d'émettre l'hypothèse que cette variable pourrait influencer positivement l'adoption des techniques agricoles et des variétés améliorées.

\subsection{Méthode d'analyse}

\section{Analyses statistiques}

Des régressions logistiques ont été effectuées pour déterminer les facteurs qui influencent l'adoption des technologies.

\section{Les modèles empirique et conceptuel}

Le modèle Logit, souvent utilisé dans les études d'adoption des technologies comme celles de Adésina et al. (2000) et de Sale et al. (2014) a été retenu dans le cadre de cette étude. Il est expliqué à travers celui-ci des phénomènes dont les manifestations ne peuvent prendre que deux valeurs 0 et 1 . Dans la littérature trois types de modèles sont principalement utilisés pour analyser la décision des producteurs à adopter une technologie agricole : les modèles de probabilité linéaire, de LOGIT et de PROBIT . Le premier modèle présente des inconvénients parce que la probabilité peut souvent dépasser 1. Les deux derniers modèles sont les plus couramment utilisés pour spécifier les relations entre la probabilité de choix et les variables déterminantes du choix (CIMMYT, 1993). Le LOGIT a l'avantage de faciliter l'interprétation des paramètres $\beta$ associées aux variables explicatifs xi (Amemiya, 1981). La fonction de répartition $\mathrm{F}(\mathrm{w})$ correspond à la fonction logistique $\forall \mathrm{w} \in \mathrm{R}$ :

$$
F(w)=\frac{e^{w}}{1+e^{w}}=\frac{1}{1+e^{-w}} \Delta(w)
$$

Ce modèle définit la probabilité associée à l'événement yi $=1$, comme la valeur de la fonction de répartition de la loi logistique considérée au point xi $\beta$ :

$$
P i=\Delta\left(x_{i} \beta\right)=\frac{1}{1+e^{-\left(\beta 0+\beta i^{Z}\right)}} \forall i=1 \ldots . N
$$

Avec :

$W=\beta 0+\beta 1$ COMMUNE $+\beta 2$ SEXE $+\beta 3 A G E+\beta 4 S I T U M A T+\beta 5$ NIVINSTR $+\beta 6 A P P O P+\beta 7 C O N T A C A G R I+\beta 8$ ACCREDIT $+\beta 9$ DVILGVLE $+\beta 10$ DMRCHPRCH + $\beta 11$ FORMATION $+e i$ 
Avec :

Où $\beta 0$ est le terme constant ; $\beta$ i les coefficients à estimer et ei les termes d'erreur.

Le logiciel SPSS 20 a été utilisé pour les statistiques descriptives et les régressions économétriques. L'index de détermination des taux d'adoption est calculé par le rapport du nombre de producteurs utilisateurs de la pratique concernée sur le nombre total des enquêtés, rapporté à 100. Les taux d'adoption représentent ainsi les proportions des paysans utilisateurs de la pratique.

Tableau 1 : les variables et les effets attendus

\begin{tabular}{|c|c|c|}
\hline Variables et codes & Description & Effets attendus \\
\hline \multicolumn{3}{|l|}{$\begin{array}{l}\text { Variable } \\
\text { dépendante }\end{array}$} \\
\hline ADOPTION & 1 si oui, 0 si non & \\
\hline \multicolumn{3}{|l|}{$\begin{array}{c}\text { Variable } \\
\text { explicatives }\end{array}$} \\
\hline AGE : & Age de l’enquêté (en année) & + \\
\hline SEXE : & 1 homme ; 0 femme & \pm \\
\hline SITUMAT & 1 mariés ; 0 si non & \\
\hline CHARG : & Nombre de personnes du ménage & + \\
\hline NIVENSTR : & $\begin{array}{l}\text { Niveau de d'instruction des répondants : } 1 \text { instruit ; } 0 \\
\text { non instruit }\end{array}$ & + \\
\hline CONTACAGRI & $\begin{array}{l}1 \text { si le producteur a été apprenant CEP, et a bénéficié } \\
\text { du soutien technique et } 0 \text { SI non }\end{array}$ & + \\
\hline ACCREDIT : & Accès au crédit 1 oui, 0 non & \pm \\
\hline APPOP & 1 si membre, 0 si non & \\
\hline DVILGVLE & $\begin{array}{c}1 \text { pour les villages proches de la ville }(<10 \mathrm{~km}) \text { et avec } \\
\text { une route facilitant l'accès, et } 0 \text { pour les villages } \\
\text { éloignés. }\end{array}$ & + \\
\hline DMRCHPRCH & $\begin{array}{l}1 \text { pour les villages proches d’un marché périodique } \\
(<10 \mathrm{~km}) \text { et avec une route facilitant l'accès, et } 0 \text { pour } \\
\text { les villages enclavés. }\end{array}$ & + \\
\hline FORMATION & $\begin{array}{l}1 \text { si le producteur a été apprenant champ } \\
\text { démonstration, et a bénéficié du soutien technique et } 0 \\
\text { SI non }\end{array}$ & + \\
\hline
\end{tabular}

\section{Résultats}

\subsection{Caractéristiques sociodémographiques des exploitants}

Cette enquête a concerné un échantillon de 120 producteurs dont 66,7\% d'hommes 33,3 \% des femmes. Ils sont âgés de 30 à 65 ans avec une moyenne de 45,33 $\pm 11,00$ ans. Quatre-vingt-quinze pourcent (95\%) d'entre eux sont mariés avec une taille de famille moyenne de 12,4 $\pm 5,5$ personnes à charge. Le nombre moyen d'actifs agricoles au sein des ménages est de 8,03 \pm 0,43 personnes. Seulement 29,66\% des enquêtés sont scolarisés dont 19,49\% ont un niveau primaire et $10,17 \%$ un niveau secondaire. Aussi, 35,59\% des producteurs sont alphabétisés en langue française mais 34,75\% sont encore des 
analphabètes. L'agriculture et l'élevage constituent leurs principales activités et $26 \%$ d'entre eux ont accès au crédit agricole

\subsection{Taux d'adoption des technologies diffusées}

Selon les communes, les taux d'adoption des technologies améliorées diffusées varient de 9,83 à $86,43 \%$ sans distinction des formés et des non formés (tableau 2). Les technologies à plus grand taux sont les variétés améliorées et la fumure organique. Pour les variétés améliorées, le mil (HKP) a un taux d'adoption plus important que le niébé (IT 90K 372-1-2).

Tableau 2 : Taux d'adoption des technologies améliorées de production du mil et du niébé dans quatre communes du département de Mayahi

\begin{tabular}{|c|c|c|c|c|c|c|c|c|}
\hline $\begin{array}{c}\text { Groupe } \\
\text { de } \\
\text { variables }\end{array}$ & Variables & Attantané & Mayahi & $\begin{array}{l}\text { Sherkin } \\
\text { Haoussa }\end{array}$ & Tchaké & \%Moyen & $\begin{array}{c}\chi^{2} \\
\text { (2d.f) }\end{array}$ & Seuil \\
\hline \multirow{2}{*}{$\begin{array}{c}\text { Variété } \\
\text { améliorées }\end{array}$} & Mil(HKP) & 90,00 & 89,66 & 93,33 & 72,73 & 86,43 & 4,39 & ns \\
\hline & Niébé(IT90) & 85,00 & 79,31 & 81,67 & 81,82 & 81,95 & 0,25 & ns \\
\hline \multirow{5}{*}{$\begin{array}{l}\text { Gestion de } \\
\text { la fertilité } \\
\text { des sols }\end{array}$} & $\begin{array}{c}\text { Fumure } \\
\text { organique }\end{array}$ & 95,00 & 86,21 & 81,67 & 81,82 & 86,18 & 2,21 & ns \\
\hline & Compost & 40,00 & 44,83 & 45,00 & 36,36 & 41,55 & 0,40 & ns \\
\hline & $\begin{array}{l}\text { Défrichement } \\
\text { amélioré }\end{array}$ & 40,00 & 55,17 & 31,67 & 45,45 & 43,07 & 4,65 & ns \\
\hline & Zaï & 10,00 & 20,69 & 13,33 & 27,27 & 17,82 & 2,38 & ns \\
\hline & $\begin{array}{l}\text { Labour } \\
\text { profond }\end{array}$ & 5,00 & 6,90 & 18,33 & 9,09 & 9,83 & 3,84 & ns \\
\hline \multirow{3}{*}{$\begin{array}{c}\text { Gestion } \\
\text { des } \\
\text { ravageurs }\end{array}$} & Sacs PICS & 55,00 & 51,72 & 53,33 & 63,64 & 55,92 & 0,48 & ns \\
\hline & Bio pesticide & 55,00 & 27,59 & 36,67 & 54,55 & 43,45 & 4,98 & ns \\
\hline & $\begin{array}{c}\text { Lutte } \\
\text { biologique }\end{array}$ & 30,00 & 24,14 & 33,33 & 54,55 & 35,51 & 3,43 & ns \\
\hline $\begin{array}{l}\text { Aliment } \\
\text { bétail }\end{array}$ & $\begin{array}{c}\text { Achat } \\
\text { BMND }\end{array}$ & 70,00 & 62,07 & 37,29 & 54,55 & 55,98 & 8,78 & $* *$ \\
\hline
\end{tabular}

Les taux d'adoption calculés par technologie ont varié de 10,1 à 95,71\% pour les producteurs formés et entre 9,55 et $88 \%$ pour les non formés. Les ratios sont 0,95 à 3 fois plus élevés pour les 11 technologies recensées dans le cas des producteurs formés par rapport à ceux non formés. Ces différences sont significatives avec les variétés améliorées des deux spéculations, le compostage, le bio pesticide, la conservation du niébé avec le sac PICS et l'utilisation des sacs de lâcher pour la lutte contre la chenille mineuse de l'épi du mil (tableau 3). 
Tableau 3 : Taux d’adoption des technologies améliorées de production du niébé et mil pour les producteurs formés et non formés à travers le champ de démonstration

\begin{tabular}{|c|c|c|c|c|c|c|}
\hline Groupe & Variables & Non formés & Formés & \%Moyen & $\chi^{2}$ (2d.f) & seuil \\
\hline \multirow{2}{*}{$\begin{array}{c}\text { Variétés } \\
\text { améliorées }\end{array}$} & Mil(HKP) & 77,15 & 95,71 & 86,43 & 6,19 & $* *$ \\
\hline & Niébé(IT90) & 70,5 & 93,4 & 81,95 & 7,79 & $* *$ \\
\hline \multirow{5}{*}{$\begin{array}{l}\text { Gestion de la } \\
\text { fertilité }\end{array}$} & Fumure organique & 88,00 & 84,35 & 86,18 & 0,6 & ns \\
\hline & Compost & 20,00 & 63,10 & 41,55 & 19 & $* * *$ \\
\hline & Défrichement amélioré & 42,13 & 44 & 43,07 & 0,57 & ns \\
\hline & Zaï & 15,63 & 20,00 & 17,82 & 2,18 & ns \\
\hline & Labour profond & 09,55 & 10,1 & 9,83 & 0,4 & ns \\
\hline \multirow{3}{*}{$\begin{array}{l}\text { Gestion des } \\
\text { ravageurs }\end{array}$} & Sacs PICS & 31,00 & 80,84 & 55,82 & 20,16 & $* * *$ \\
\hline & Biopesticide (Neem) & 22,9 & 64,00 & 43,45 & 16,11 & $* * *$ \\
\hline & Lutte biologique & 22,02 & 49,00 & 35,51 & 4,3 & $* *$ \\
\hline $\begin{array}{c}\text { Aliment } \\
\text { bétail }\end{array}$ & Achat BMND & 50,96 & 60,99 & 55,98 & 1,9 & ns \\
\hline
\end{tabular}

(Enquête, 2016)

${ }^{* * *} \mathrm{p}<0,001 \quad * * \mathrm{p}<0,05 \quad$ ns : différence non significative

\subsection{Facteurs déterminant l'adoption des technologies}

Il a été utilisé au total 11 facteurs susceptibles d'influencer l'adoption des technologies dans l'étude avec le modèle LOGIT, mais 6 de ces variables ont eu une influence significative sur l'adoption de certaines des technologies (Tableau 4) :

- l'appartenance à un champ de démonstration initié par le projet (Formation) a influencé positivement l'adoption des technologies suivantes par ordre d'importance : 1) L'application du compost, 2) l'utilisation de la variété améliorée du mil (HKP), 3) l'utilisation des Sacs PICS, 4) l'utilisation des extraits aqueux des graines de Neem et 5) l'utilisation de la variété améliorée du niébé (IT90K 372-1-2) ;

- la proximité des villages au marché a négativement influé sur l'utilisation du compost ;

- La proximité des villages au département de Mayahi a influencé positivement 1) l'achat des blocs multi nutritionnels densifiés (BMND) et 2) la pratique du défrichement amélioré ;

- l'accès au crédit (ACCREDIT) a donné une corrélation positive avec l'achat des blocs multi nutritionnels densifiés (BMND) ;

- le contact permanent avec la Direction Départementale de l'Agriculture (DDA) a influencé positivement l'utilisation des sacs de lâcher pour la lutte contre la chenille mineuse de l'épi du mil ; et puis

la situation matrimoniale autrement dit le mariage a influencé positivement, l'achat des BMND. 
ESJ Natural/Life/Medical Sciences

Tableau 4 : Résultat du modèle d'estimation des facteurs d'adoption des technologies améliorées

\begin{tabular}{|c|c|c|c|c|c|c|c|c|c|c|c|}
\hline Variables & Mil(HKP) & $\begin{array}{l}\text { Niébé } \\
\text { (IT90) }\end{array}$ & $\begin{array}{c}\text { Défrichement } \\
\text { Amélioré }\end{array}$ & FO & $\begin{array}{c}\text { Comp } \\
\text { ost }\end{array}$ & Zaï & $\begin{array}{l}\text { Labour } \\
\text { profond }\end{array}$ & $\begin{array}{c}\text { Biopesticid } \\
\text { e } \\
\text { (Neem) }\end{array}$ & $\begin{array}{c}\text { Lutte } \\
\text { biologiq } \\
\text { ue }\end{array}$ & $\begin{array}{l}\text { Sacs } \\
\text { PICS }\end{array}$ & $\begin{array}{l}\text { Achat } \\
\text { BMD }\end{array}$ \\
\hline Comm & 1,29 & $-0,06$ & 0,17 & $-0,88$ & $-0,43$ & $-0,78$ & 0,93 & $-0,78$ & $-0,43$ & $-0,60$ & $-0,82$ \\
\hline Sexe & 0,08 & $-0,01$ & $-0,15$ & 0,66 & $-0,14$ & 0,03 & 1,11 & 0,05 & 0,38 & 0,07 & $-0,44$ \\
\hline Age & $-0,52$ & $-0,77$ & 0,20 & $-0,09$ & $-0,20$ & $-0,63$ & 0,22 & $-0,17$ & $-0,20$ & $-0,84$ & $-0,38$ \\
\hline Situmat & $-19,44$ & $-20,75$ & 1,79 & 0,43 & $-0,20$ & $-0,02$ & $-0,81$ & $-1,07$ & 0,69 & $-0,81$ & $2,65^{* *}$ \\
\hline Nivinstr & $-0,18$ & $-0,39$ & 0,02 & 0,53 & 0,22 & $-0,26$ & 0,38 & 0,76 & $-0,34$ & 0,67 & $-0,29$ \\
\hline АррОР & 1,64 & 0,13 & 0,97 & 0,38 & 0,12 & 0,23 & 0,32 & $-0,27$ & $-0,51$ & 0,42 & 0,94 \\
\hline ContactAgri & $-0,42$ & 1,18 & $-0,75$ & 1,06 & 0,09 & $-0,61$ & 0,70 & 1,14 & $1,87 * *$ & 0,23 & $-1,43^{* *}$ \\
\hline Accredit & $-1,04$ & 0,38 & 0,97 & 0,06 & $-0,02$ & $-1,01$ & $-0,58$ & $-0,02$ & 0,42 & $-0,39$ & $1,11^{* *}$ \\
\hline Dvilgvle & 0,34 & $-0,82$ & $2,08 * * *$ & 0,05 & 0,35 & 0,39 & $-1,28$ & $-0,02$ & $-0,28$ & 0,24 & $2,53^{* * *}$ \\
\hline Dmrchprch & $-0,17$ & 0,63 & $-0,04$ & $-0,18$ & $-1,62^{* *}$ & $-1,46$ & 0,22 & 0,54 & $-0,01$ & $-0,76$ & $-0,50$ \\
\hline Formation & $1,85^{* *}$ & $1,15^{* *}$ & $-0,13$ & $-0,88$ & $\begin{array}{c}1,93^{* *} \\
*\end{array}$ & 1,24 & 0,62 & $1,66 * * *$ & 0,55 & $1,79 * * *$ & 0,24 \\
\hline Constant & 20,03 & 21,44 & $-3,83$ & 1,20 & $-0,30$ & $-0,42$ & $-3,44$ & $-1,11$ & $-2,64$ & 0,57 & $-2,46$ \\
\hline
\end{tabular}


ESJ Natural/Life/Medical Sciences

\section{Discussion}

A l'issue de cette étude, des renseignements sur l'adoption des technologies mise en œuvre depuis 2014 par le projet d'appui à la sécurité alimentaire par l'intensification agro-écologique dans le département de Mayahi ont été documentés.

En ce qui concerne les variétés améliorées du mil (HKP) et du niébé (IT 90K 372-1-2), le taux moyen d'adoption est respectivement de $82 \%$ et $70 \%$ pour les producteurs non formés puis 95,71 et $90 \%$ pour les formés. Il est bien constaté que le taux d'adoption de la variété améliorée du niébé est largement supérieur à celui enregistré par Rabé et al. (2017) qui ont trouvé un taux d'adoption de la variété IT 90K 372-1-2 de 55\% chez les producteurs non formés et 67,3\% dans le groupe des producteurs ayant participé aux CEP. Ce qui illustre une tendance similaire entre formation et adoption des variétés améliorées de mil et niébé. Le taux élevé d'adoption des variétés améliorées peut aussi s'expliquer par le potentiel de rendement de ces variétés (Dugje et al., 2009 ; Saidou 2011). Cela confirme aussi les affirmations de Bellon et al., (2006) qui ont avancé que les producteurs adoptent les variétés améliorées afin de maximiser les avantages des caractéristiques tels que le rendement, la résistance aux maladies. On constate par ailleurs que les taux d'adoption des variétés améliorées sont très élevés aussi bien chez les producteurs formés que chez les producteurs non formés. Autrement dit, les producteurs ont tendance à adopter les variétés du mil et du niébé dans le département de Mayahi. Selon une étude réalisée dans le département de Mayahi, le rendement de la variété HKP a été remarquable (1030 Kg/ha) [PASADEM \& DRA, 2014]. Cela peut expliquer cet engouement des producteurs à son adoption.

Pour le redressement de la fertilité du sol, le compostage, la fumure organique et le zaï ont été proposés aux producteurs. En effet, la pauvreté des sols constitue une contrainte permanente en milieu paysan sahélien (Osbahr, \& Allan, 2003 ; Dutordoir, 2006). Mais le zaï (15,83\%) et le compostage (43,33\%) ont été les moins adoptés. Le taux d'adoption de ces technologies est par ordre respectif de 2 à 3 fois plus élevé dans le groupe des producteurs formés. En effet, les probabilités d'adoption des techniques de conservation des eaux et des sols sont faibles si les producteurs n'en tirent pas un bénéfice à court terme (Zoungrana, 2004).

L'adoption des fertilisants (compost) est significativement influencée par la formation reçue dans les champs de démonstration. La pratique de compostage est l'une des technologies la plus développée dans les champs de démonstrations et cela explique les taux d'adoption plus élevés au niveau des producteurs adhérant aux processus. 
L'accès aux ressources financières telles que le crédit explique l'achat des BMD. Ce résultat est similaire à celui de Sale et al. (2014) qui indiquent que l'accès au crédit est déterminant pour le choix d'une stratégie, car les producteurs ayant un accès facile aux crédits auront tendance à mieux adopter les nouvelles technologies.

Pour la lutte contre les ravageurs des cultures du niébé, il est noté un taux d'adoption du bio pesticide pour les producteurs formés 3 fois plus élevé que celui des non formés. Tout naturellement, l'acquisition d'informations nouvelles chez les producteurs justifie la différence du taux d'adoption du biopesticide. L'influence positive de la formation sur l'utilisation des méthodes alternatives non chimiques a été déjà démontrée par Djinadou et al. (2008) puis Rabé et al. (2017). Bien que l'expression de leur potentiel de rendement soit conditionnée par une bonne gestion des ravageurs (Oyewale et al., 2014), le mil et le niébé sont des cultures bien adaptées aux sols sahéliens arides.

Le taux moyen d'adoption des biopesticides à base de graines de neem est de 39,17\% pour les deux groupes de producteurs. Ce taux est nettement supérieur à celui enregistré par Adéoti et al. (2002) puis Rabé et al. (2017) dans la région de Maradi. Cette situation peut s'expliquer par le fait que le biopesticide proposé aux producteurs est un produit fini alors que pour les précédents le travail fastidieux de préparation de jus de grains de neem a été l'œuvre des concernés. Ce qui confirme encore les raisons de pénibilité liée à l'utilisation de cette technologie déjà évoquées par Adéoti et al. (2002).

En ce qui concerne l'achat des sacs de lâcher pour la lutte contre la mineuse de l'épi du mil, le taux d'adoption pour les producteurs formés est 2 fois plus élevé comparé aux non formés. Ce qui explique l'influence positive du champ de démonstration sur l'utilisation des méthodes alternatives non chimiques. Selon Khonje et al. (2014), pour les formations des producteurs, l'accès aux services de vulgarisation, la diffusion des informations sur le marché font parties des principaux facteurs déterminant l'adoption des technologies agricoles.

La pression démographique ainsi que l'irrégularité pluviométrique causée par la sècheresse récurrente au sahel ont entraîné, une dégradation des ressources naturelles. Des études faites dans le département de Maradi (Niger) par le CNEARC et la Faculté d'agronomie de Niamey ont permis de caractériser une dégradation des ressources arbustives et arborées et d'en analyser les conséquences sur les systèmes de production de la région. Pour faire face à cette rareté des ressources naturelles, il a été mis à la disposition des producteurs la technologie de défrichement améliorée. Cette pratique est déjà adoptée puis maîtrisée par $40 \%$ des producteurs toute catégorie confondue. Ce qui met en exergue un changement de comportement de la population cible dans la gestion des ressources. Ce changement est beaucoup plus senti au niveau des villages qui sont à proximité du département de 
Mayahi. Il est donc à déduire que les enjeux de la dégradation des ressources naturelles sont plus perceptibles par les producteurs de ces contrées.

La formation et la sensibilisation des producteurs sur les sacs PICS ont été déterminantes dans l'adoption de cette technologie. Par contre, d'autres variables a priori pertinentes présentes dans le modèle n'ont pas eu d'effet significatif sur cette adoption. Il s'agit notamment de l'âge, du sexe, du statut matrimonial, du niveau d'instruction, et de l'appartenance à l'organisation des producteurs. Cette situation peut s'expliquer par l'intensité et la pertinence des formations mais aussi par la simplicité de la technologie.

\section{Conclusion}

Les facteurs déterminants l'adoption des technologies de production du mil, du niébé et de l'achat des blocs multi nutritionnels densifiés sont variables d'une technologie à une autre. La plupart de ces facteurs sont socioéconomiques et techniques puis sont non seulement liés aux producteurs mais aussi à la non complexité des technologies.

Le processus de champ de démonstration a été significativement déterminant sur le taux d'utilisation des technologies diffusées. Les résultats de l'étude d'adoption des technologies agroécologiques diffusées par le canal de l'approche champ de démonstration ont permis de connaitre les facteurs sur lesquels il faut se baser pour avoir un taux d'adoption acceptable.

Ces taux concernent l'utilisation des variétés améliorées, du compost, la gestion des ravageurs des cultures, la lutte biologique, l'utilisation des sacs PICS qui sont des éléments déterminants pour l'augmentation de la production.

En ce qui concerne les variétés améliorées, il est important de poursuivre la promotion d'autres variétés et le renforcement des réseaux de production des semences pour fournir aux producteurs un choix varié.

Le taux d'acheteurs des blocs multi nutritionnels densifiés (BMND) est lié à la proximité du lieu de vente, mais aussi à l'accès au crédit. Il semble important de développer le crédit et aussi de renforcer les réseaux de commercialisation des BMND. Il faut poursuivre la promotion d'autres technologies agro-écologiques avec l'approche champ de démonstration et le renforcement des réseaux de diffusion de ces technologies. 


\section{References:}

1. Adesina, A. A., Mbila, D., Nkamleu, G. B., \& Endamana, D. (2000). Econometric analysis of the determinants of adoption of alley farming by farmers in the forest zone of southwest Cameroon. Agriculture, Ecosystems \& Environment, 80(3), 255-265.

2. Adeoti R, Coulibaly O., \& M Tamo. (2002). Facteurs affectant l'adoption des nouvelles technologies du niébé Vigna unguiculata en Afrique de l'Ouest. Bull. Rech. Agron. Benin, 36 ,18-26.

3. Banque Mondiale. (2010). Rapport annuel : bilan de l'exercice. 37pp. http://siteresources.

worldbank.org/EXTANNREP2010FRE/Ressources/Banque mondiale.Rapport annuel(2010).pdf Consulté le 27 décembre 2016

4.Barmo S., Amani A., Sitou L., Ichaou A., Ali M., \& Mahamane S., (2017). Typologie des exploitations agricoles familiales : cas de la périphérie de la forêt protégée de Baban Rafi du Niger. Int. J. Biol. Chem. Sci. 11(3): 1096-1112, June 2017. ISSN 1997-342X (Online), ISSN 1991-8631 (Print). http://ajol.info/index.php/ijbcs

5. Dey J. (1981). Gambian Women: unequal partners in rice development projects. The Journal of Development Studies. 17 (3), 143-151.

6. Djinadou KA, Coulibaly O., Agbo VA., \& Adégbidi AA. (2008a). Genre et accès aux facteurs de production pour l'adoption des extraits aqueux de Neem (Azadirachta indica) au Bénin. Les Cahiers de l'Economie Rurale, 6 : 69-80.

7..Dugje I.Y., Omoigui L.O., Ekeleme F., Kamara A.Y., \& H., Ajeigbe. (2009). Production du niébé en Afrique de l’Ouest : Guide du paysan. IITA, Ibadan, Nigeria. 20p.

8. .Dutordoir CD. (2006). Impact de pratiques de gestion de la fertilité sur les rendements en mil dans le Fakara (Niger). Travail de fin d'études présenté en vue de l'obtention du grade de bio-ingénieur.Retrouvé le 18/11/2016 sur le web à l'adresse: https://www.jircas.affrc.go.jp/ project/africa_dojo/Metadata/grad_research/03.pdf

9..Ibro G., \& Bokar M. (2001). Transfert des nouvelles technologies dans le système de production des paysans au Niger. Etude de cas : adoption des variétés améliorées et de nouvelles techniques de production de la culture du niébé. Rapport soumis à l'IITA, projet PEDUNE, 17p. Retrouvé le 18/11/2016 à l'adresse http://www.fidafrique.net/ IMG/pdf/Technology_adoption_Pronaf_Niger.pdf.

10. 10.Jama B., \& Gonzalo P. (2008). Agriculture in Africa: Strategies to Improve and Sustain Smallholder Production Systems. Ann. N.Y. Acad. Sci. New York 1136, 218-232

11. .Khonje M, Manda J., \& Alene AD, Kassie M.(2014). Analysis of Adoption and Impacts of Improved Maize Varieties in Eastern Zambia. 
World Development. 66, 696-706. DOI: http://dx.doi.org/10.1016/j.worlddev.2014.09.008

12. .MAG-SDR. (2010). Etude sur la mise en place d'un dispositif intégré d'appui conseil pour le développement rural au Niger. Etude sur la mise en place d'un dispositif intégré d'appui conseil pour le développement rural au Niger. Secrétariat exécutif comité interministériel de pilotage de la stratégie de développement rural, $127 p$.

13. MA. (2015). Résultats définitifs de la campagne agricole 2014 ; perspectives alimentaires 2014-2015: Ministère de l'agriculture. Direction des statistiques. 32p. Retrouvé sur le web le 04/11/2016 à l'adresse :

http://www.recaniger.org/IMG/pdf/Resultats_definitifs_Campagne_agricole_2014.pd $\mathrm{f}$

14. Oyewale RO, Bamaiyi LJ, Oparaeke AM., \& Adamu R S. (2014 à. Evaluation of four insecticide formulations for the management of insect pests of cowpea, Journal of Food Science and Technology., 5(8), 180-188.

15. Osbahr H., \& Allan C. (2003). Indigenous knowledge of soil fertility management in southwest Niger. Geoderma, 111(3), 457-479. DOI: http://dx.doi.org/10.1016/S0016-7061(02)00277-X9.

16. Rabé, M.M., Baoua I., Adeoti, R., Sitou, L., Amadou, L., \& Pittendrigh, B., (2017). Les déterminants socioéconomiques de l'adoption des technologies améliorées de production du niébé diffusées par les champs écoles paysans dans les régions de Maradi et Zinder au Niger. International Journal of Biological and Chemical Sciences, 11(2), 744 $-756$.

DOI https://dx.doi.org/10.4314/ijbcs.v11i2.17

17. Rabé, M.M., Baoua, I.B., Sitou, L., \& Amadou, L., (2017a). Champ école paysan, une approche participative pour l'amélioration du rendement du niébé : résultats d'expériences pilotes conduites dans les régions de Maradi et Zinder au Niger. Agronomie Africaine, 29(2), 19. DOI : https://www.ajol.info/index.php/aga/article/view/163178

18. Renard JF, Cheikh L, Knips V. (2004). L'élevage et l'intégration régionale en Afrique de l'Ouest, Ministère des Affaires étrangères FAO-CIRAD, 37p.

19. Sermé I, Outtara K, Logah V, Taounda JB, Pale S, Quansah C., \& Abaidoo R. (2015).Impact of tillage and fertility management options on selected soil physical properties and sorghum yield. International Journal of Biological and Chemical Sciences. 9(3), 1154- 1170. doi : http://dx.doi.org/10.4314/ijbcs.v9i3.2 
20. Sanginga PC, Adesina AA, Manyong VM, Otite O., \& Dashiell K E. (1999). Social impact of soybean in Nigeria's southern Guinea savanna. International Institute of Tropical Agriculture. 34 p. Retrouvé sur le web le 18/11/2016 à l'adresse : http://www.tropicalsoybean.com/sites/default/files/Social\%20Impact \%20Of\%20Soybean\%20In\%20Nigeria\%27S\%20Southern\%20Guine a\%20Savanna_Sanginga\%2C\%201999.pdf

21. Sani R. M., \& Bagna A. (2007). Adoption of improved seed s of millet and cowpea by farmers in Madarounfa district, Niger republic. Continental Journal of Agricultural Economics 1: 1-6. Saxena R. C. and E. L. Kidiavai.1997. Neem seed extract spray applications as lowcost inputs for management of the flower thrips in the cowpea crop. Phytoparasitica, 25 (2): 99-110.

22. Sale A, Folefack PD, Obwoyere GO, Lenah Wati N, Lendzemo WV., \& Wakponou A. (2014). Changements climatiques et déterminants d'adoption de la fumure organique dans la région semi-aride de Kibwezi au Kenya. International Journal of Biological and Chemical Sciences 8(2), 680-694. doi : http://dx.doi.org/10.4314/ijbcs.v8i2.24

23. Saidou AK, Ajeigbe HA., \& BB Singh. (2011). Participatory Evaluation of Improved Cowpea Lines and Cropping Systems for Enhancing Food Security and Income Generation in Niger Republic, West Africa. American-Eurasian Journal of Agriculture and Environmental Science, 11, 55-61.

24. Sherin, S. P., N, Mathew, P, Johns, F., \& Abraham, J. (2018). The feasibility of using remote data collection tools in field surveys. International Journal of Community Medicine and Public Health. 5, 81-5.

25. Zoungrana C., (2004). Adoption des techniques de conservation des sols et lutte contre la pauvreté au Yatenga. Mémoire de D.E.A. Université de Ouagadougou, UFR/SEG, 76p 